\title{
USING SIMULATION TO ASSESS COSTS OF QUALITY
}

\author{
L. Leslie Gardner \\ University of Indianapolis \\ 1400 East Hanna Avenue \\ Indianapolis, IN 46227 U.S.A.
}

\author{
Mary E. Grant \\ Pritsker Corporation \\ 8910 Purdue Road, Suite 500 \\ Indianapolis, IN 46268 U.S.A.
}

\author{
Laurie J. Rolston \\ DowElanco \\ 9410 Zionsville Road \\ Indianapolis, IN 46268 U.S.A.
}

\begin{abstract}
This paper illustrates the impact of defective rates and inspection and defective removal strategies on the profitability of a manufacturing system. The use of simulation to assess cost of quality for justification of quality improvement is demonstrated.
\end{abstract}

\section{INTRODUCTION}

Improving product quality can give a company a tremendous competitive advantage and greatly enhance profitability. An effort to improve quality may be justified by comparing the costs of failure to implement with the costs of implementation. Costs of quality are categorized as:

- Failure costs -- internal and external

- Appraisal costs

- Prevention costs

Internal failure costs are the cost of manufacturing defective parts or products that cannot be sold, disposal of scrap, and rework. External failure costs include liability, and lost customers. Appraisal costs are primarily the costs of inspection and testing. Prevention costs encompass planning and design, working with vendors, training, and a variety of quality control procedures (Campanella, 1990).

Simulation can be used to assess the impact of defective rates of each operation on the overall profitability and productivity of the manufacturing system. Models can be constructed of the "as is" manufacturing system and of the system after proposed improvements and defective rate reductions are made. Performance measures obtained from these simulations can be converted to manufacturing costs and revenue and the profitability of each situation compared (Gardner, Grant, and Rolston, 1992). Cost of quality is the difference in profitability between the "as is" and the improved situation. A quality improvement effort can be justified if the increase in profitability is sufficient to cover the costs of implementation.
Simulation is flexible enough to model a wide variety of issues that arise with regard to quality and manufacturing. Defective rates need not be constant. Simulation allows modeling of defective rates that change over time and depend on setups, product mix, time in queue, time since the last preventive maintenance, and other factors. Inspection points, acceptance sampling, and removal of defectives can also be modeled, allowing the effectiveness of the placement of inspection points and sampling strategies to be tested.

Passing defective parts through a manufacturing system generates cost in terms of wasted labor and machine time, wasted materials, and wasted energy. Inspection and removal of defectives also consumes time and resources, and may even generate waste if the inspection and testing process is destructive. Defective parts should be removed from the system whenever the cost of passing defectives exceeds the cost of inspection. Inspection cost can be cut by the use of acceptance sampling at the risk of passing some defectives. The model of paper illustrates the impact on profitability and productivity of four strategies for inspection and removal of defectives across a range of defective rates:

1. Inspection and removal of defectives at completion of finished product only,

2. Inspection and removal of defectives prior to assembly points,

3. Inspection and removal of defectives following every operation, and

4. Inspection and removal of defectives based on acceptance sampling prior to assembly points.

The results are analyzed with regard to internal failure and inspection costs.

\section{THE MODEL}

The model described in this paper is a teaching model of a fictitious manufacturing system. It is designed to be complex enough to be realistic and to illustrate the variety of problems incurred in passing defectives but simple enough to keep the details from obscuring the 
principles to be tested. The model is written for the academic version of SLAMSYSTEM with Microsoft FORTRAN 5.1 inserts.

\subsection{The Manufacturing System}

To illustrate the issues associated with passing defectives, the manufacturing system had to produce a product constructed of multiple subassemblies. The bill of materials for a folding chair, which is such a product, is illustrated in Figure 1. Every item in the bill of materials has a routing with from one to seven operations. The various parts and subassemblies use fifteen resource classes representing machines and labor used in the fabrication and assembly process. Each operation for each part and subassembly has a defective rate (possibly zero) associated with it.

\subsection{Assumptions}

The model was built under the following assumptions:

1. Defective rates are binomially distributed with constant mean.

2. The defective rate at a given operation is independent of preceding operations.
3. Once a part is defective it remains defective.

4. Selection of parts for assembly is independent of whether or not they are defective.

5. If an assembly contains a defective part, the assembly is defective.

6. Inspection is not destructive but requires time.

The assumption of constant mean defective rates simplifies the modeling process. In reality, mean defective rates may change according to time since last maintenance or may be dependent on conditions such as setups and queuing. Enhancements of this model to allow mean defective rates to be a function or time and setups are planned for the future. The assumptions that defective rates are independent from operation to operation and that defective parts remain defective allow the number of good parts in a lot to be computed by simple multiplication provided no assembly takes place. The assumptions related to assemblies are key elements in the development of the code for determining whether or not assemblies are defective. The assumption that inspection is not destructive implies that lot size need not be reduced after inspection. Since additional time must be incurred for inspection, operation times are increased appropriately in the model whenever inspection and defective removal takes place.

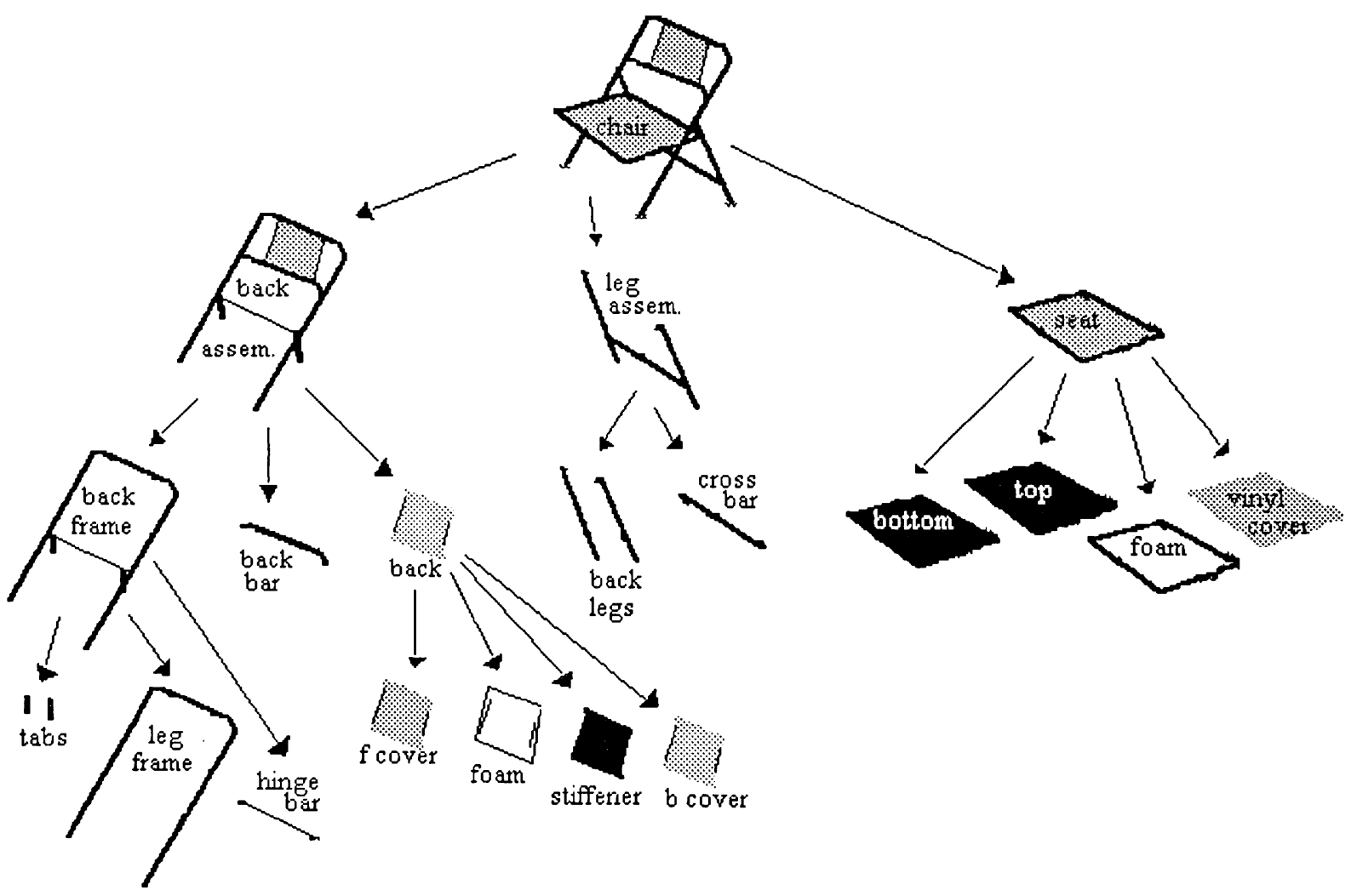

Figure 1: Bill of Materials for a Folding Chair 


\subsection{Modeling Issues}

Each simulation entity representing a lot carrics with it an attribute specifying the fraction nondefective. For each operation that is not an asscmbly, the fraction nondefective is generated by a sequence of Bernoulli trials as in the following pseudocode fragment:

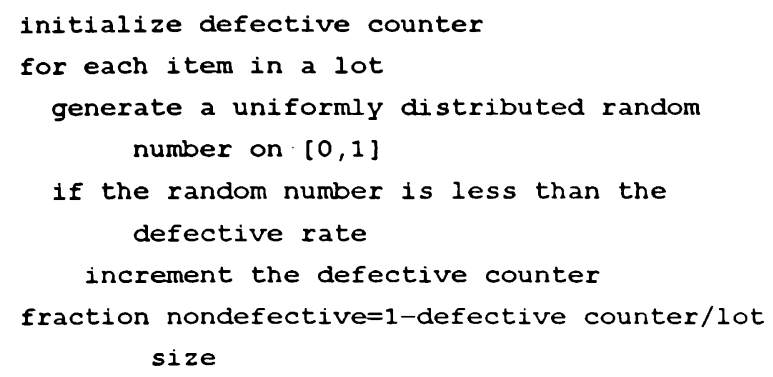

The fraction nondefective generated by the current operation is multiplied by the incoming fraction nondefective, according to the independence assumption, to give the outgoing fraction nondefective.

Obtaining the fraction nondefective of a lot at an assembly point is complex because good parts can be assembled with defective parts producing a defective assembly. Again, Bernoulli trials are employed in determining whether or not parts are defective, but the probabilities must constantly be updated as available and nondefective available parts are removed from stock. The following pseudocode fragment illustrates this process.

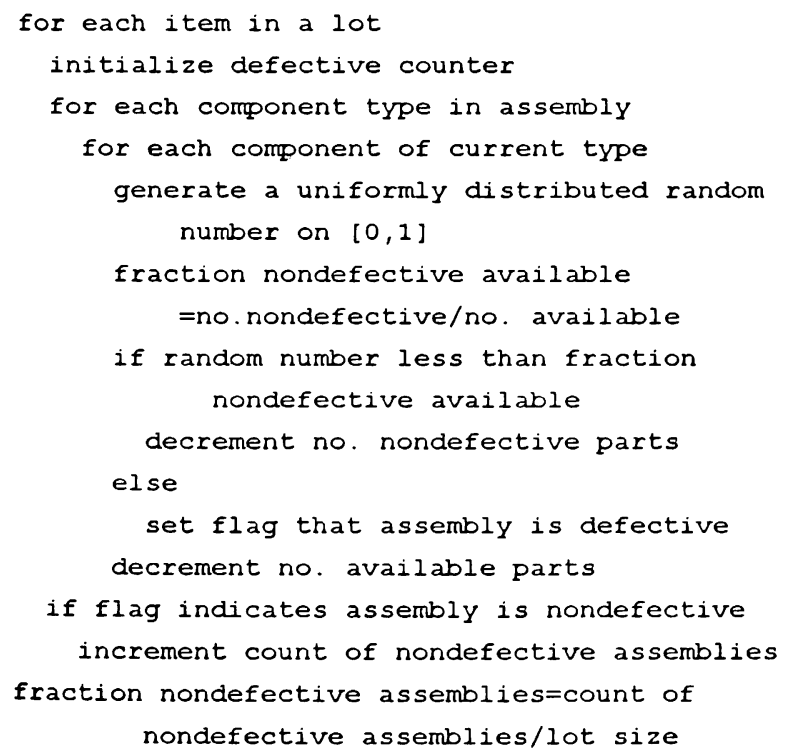

Acceptance sampling is also modeled by a sequence of Bernoulli trials with appropriate lot size and fraction nondefective changes as in this pseudocode fragment:

initialize defective counter

for each item in sample

generate a uniformly distributed random

number on $[0,1]$

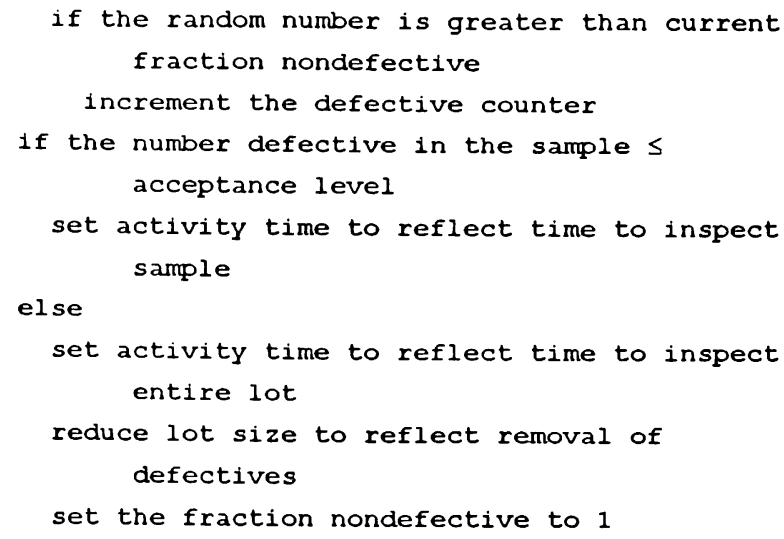

Using Bernoulli trials to model production of defective parts gives realistic variation but can greatly increase simulation run time. This is probably not a major problem because justification of improvement strategies is a design problem and the nature of design simulation is such that longer run times can often be tolerated for the sake of accuracy.

\section{THE EXPERIMENTS}

The experiments are designed to measure the impact on profitability and productivity of four strategies for inspection and removal of defectives across a spectrum of defective rates:

1. Inspection and removal of defectives at completion of finished product only,

2. Inspection and removal of defectives prior to assembly points,

3. Inspection and removal of defectives following every operation, and

4. Inspection and removal of defectives based on acceptance sampling prior to assembly points.

\subsection{Inspection and Defective Removal Strategies}

The objective of all inspection and defective removal strategies is to maximize profitability by balancing cost of inspection with cost of passing defectives. In these experiments, cost of inspection is primarily the cost of time spent inspecting product. The cost of passing defectives obtained from simulation data is internal failure cost which includes:

- time, labor, encrgy, machine wear, and material that are wasted on products that cannot be sold and do not contribute revenue, and

- the opportunity cost of product that could have been sold if it had not been defective.

Waiting until completion of the final product to inspect and remove defectives minimizes inspection cost at the expense of passing defectives. Removal of defectives after every opcration minimizes internal 
Table 1: Experimental Design

\begin{tabular}{|l|c|c|c|c|c|c|}
\hline \multirow{2}{*}{$\begin{array}{c}\text { Inspection and Defective } \\
\text { Removal Strategy }\end{array}$} & \multicolumn{5}{c|}{ Fraction Defective per Operation } \\
\cline { 2 - 7 } & $\mathbf{0 . 0 0 0}$ & $\mathbf{0 . 0 0 1}$ & $\mathbf{0 . 0 0 5}$ & $\mathbf{0 . 0 1 0}$ & $\mathbf{0 . 0 5 0}$ & $\mathbf{0 . 1 0 0}$ \\
\hline 1. Completion of finished product & 30 runs & 30 runs & 30 runs & 30 runs & 30 runs & 30 runs \\
\hline 2. Prior to asscmbly & 30 runs & 30 runs & 30 runs & 30 runs & 30 runs & 30 runs \\
\hline 3. Following crery operation & 30 runs & 30 runs & 30 runs & 30 runs & 30 runs & 30 runs \\
\hline 4. Acceptance sampling & 30 runs & 30 runs & 30 runs & 30 runs & 30 runs & 30 runs \\
\hline
\end{tabular}

failure cost. An interesting trade-off is that time spent in inspection can delay processing which in turn causes more congestion on the shop floor, late orders, and possibly overtime or an increased number of workers. On the other hand, removing defective parts can reduce the workload, help to relieve congestion on the shop floor and reduce costs of wasted resources.

Removal of defectives prior to assembly and acceptance sampling are strategies which attempt to balance such costs. Removal of defectives prior to assembly prevents the proliferation of defectives that occurs if bad parts are assembled with good. Acceptance sampling has the risk of passing lots containing more than the acceptable fraction of defectives, if by random chance, the sample selected contains an acceptably low number of defectives (type II error). Another problem is that a sample may contain a higher proportion of defectives than the lot, causing time to be wasted on $100 \%$ inspection (type I error).

\subsection{Design of Experiments}

The experiments test various inspection and defective removal strategies as summarized in Table 1. Some of the defective rates shown in the table are unrealistic in a manufacturing environment, but they make a strong impression on students for teaching purposes. To simplify the design and reporting of the cxperiments, the same defective rate is applied to every operation in the manufacturing system for a given trial. Each trial consists of 30 runs of the simulation. Each simulation run represents one year of production, that is, 250 eight hour days of regular time. For each run, 250 orders of size 100 are relcased at random intervals ranging from 0.5 day to 1.5 days. Data collected includes:

- setup and processing time for conversion to cost,

- backlog which estimates overtime, and

- throughput for conversion to revenue.

Processing times are increased by a small amount at operations where defectives are removed to reflect the time it would take for a machine operator to inspect his or her product and remove defectives. Processing times are not increased for acceplance sampling because an additional worker is modeled to do the inspection in this case. For the acceptance sampling strategy, the sample size is $10(10 \%$ of the lot size) and the lot is $100 \%$ inspected if any defectives are found. This means that the lot is rejected if $10 \%$ or more of the sample are defective. Acceptance sampling is carried out prior to every assembly and at completion of the final product.

\section{THE RESULTS}

The results of each experiment were analyzed in Microsoft EXCEL. The $90 \%$ confidence interval was less than $2 \%$ of the mean for all performance measures except volume. The $90 \%$ confidence interval for volume was the maximum of 2 units or $2 \%$ of the mean.

\subsection{Throughput}

Throughput is the quantity of nondefective final product obtained at the end of a simulation run. Throughput as a fraction of input is given in Table 2 and summarized in Figure 2. Throughput is dramatically better for strategies 2, 3, and $t$ than for strategy 1. Throughput is not significantly different for strategies 2 or 3 at any defective rate. Throughput for strategy + is also not significantly different at defective rates of $1 \%$ or greater.

\subsection{Manufacturing Costs}

Manufacturing costs are obtained from simulation data by converting

- operating time to wages,

- product volume to direct materials,

- backlog to overtime. and

- machine time to utilities and other miscellaneous costs such as tool wear, lubricant cost, and indirect materials.

Details of the conversion of simulation data to costs are described by Gardner, Grant, and Rolston (1992).

The mean manufacturing costs for each experiment are given in Table 3 and summarized in Figure 3. Manufacturing costs are relatively stable under strategy 1 , but decrease greatly as defectives are removed and fewer resources are wasted on processing defectives as in strategies 2, 3, and 4. Differences in manufacturing costs are quite pronounced for all strategies. 
Table 2: Throughput as a Fraction of Input

\begin{tabular}{|l|c|c|c|c|c|c|}
\hline \multicolumn{1}{|c|}{$\begin{array}{c}\text { Inspection and Defective } \\
\text { Removal Strategy }\end{array}$} & \multicolumn{6}{|c|}{ Defective Rate per Operation } \\
\cline { 2 - 8 } & $\mathbf{0 . 0 0 0}$ & $\mathbf{0 . 0 0 1}$ & $\mathbf{0 . 0 0 5}$ & $\mathbf{0 . 0 1 0}$ & $\mathbf{0 . 0 5 0}$ & $\mathbf{0 . 1 0 0}$ \\
\hline 1. Completion of finished product & 1.00 & 0.94 & 0.74 & 0.55 & 0.05 & 0.00 \\
\hline 2. Prior to assembly & 1.00 & 0.99 & 0.95 & 0.87 & 0.59 & 0.35 \\
\hline 3. Following every operation & 1.00 & 0.99 & 0.95 & 0.87 & 0.59 & 0.35 \\
\hline 4. Acceptance sampling & 1.00 & 0.94 & 0.91 & 0.87 & 0.59 & 0.35 \\
\hline
\end{tabular}

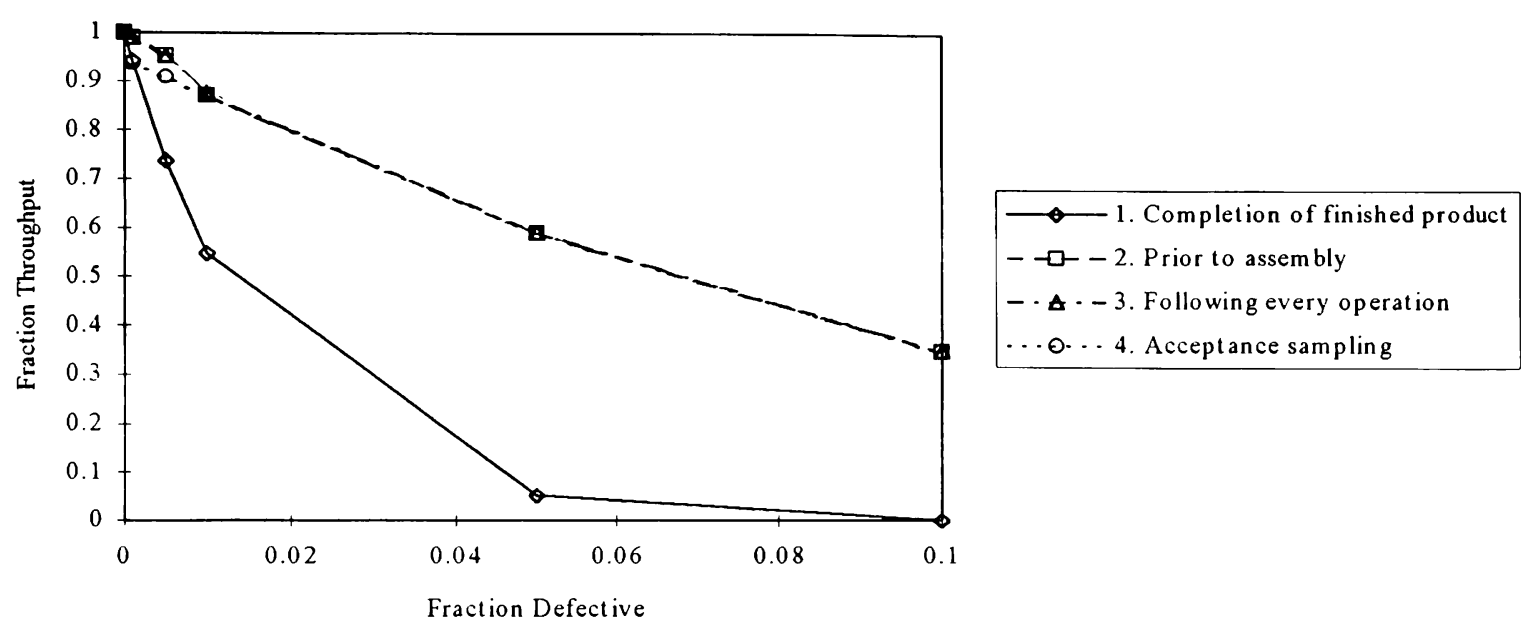

Figure 2: Throughput

Table 3: Manufacturing Costs $(\times 1000)$

\begin{tabular}{|l|c|c|c|c|c|c|}
\hline \multicolumn{1}{|c|}{$\begin{array}{c}\text { Inspection and Defective } \\
\text { Removal Strategy }\end{array}$} & \multicolumn{5}{|c|}{ Defective Rate per Operation } \\
\cline { 2 - 7 } & $\mathbf{0 . 0 0 0}$ & $\mathbf{0 . 0 0 1}$ & $\mathbf{0 . 0 0 5}$ & $\mathbf{0 . 0 1 0}$ & $\mathbf{0 . 0 5 0}$ & $\mathbf{0 . 1 0 0}$ \\
\hline 1. Completion of finished product & $\$ 661$ & $\$ 655$ & $\$ 655$ & $\$ 654$ & $\$ 650$ & $\$ 646$ \\
\hline 2. Prior to assembly & $\$ 678$ & $\$ 657$ & $\$ 641$ & $\$ 629$ & $\$ 603$ & $\$ 590$ \\
\hline 3. Following every operation & $\$ 683$ & $\$ 671$ & $\$ 660$ & $\$ 648$ & $\$ 604$ & $\$ 587$ \\
\hline 4. Acceptance sampling & $\$ 679$ & $\$ 654$ & $\$ 647$ & $\$ 641$ & $\$ 619$ & $\$ 606$ \\
\hline
\end{tabular}
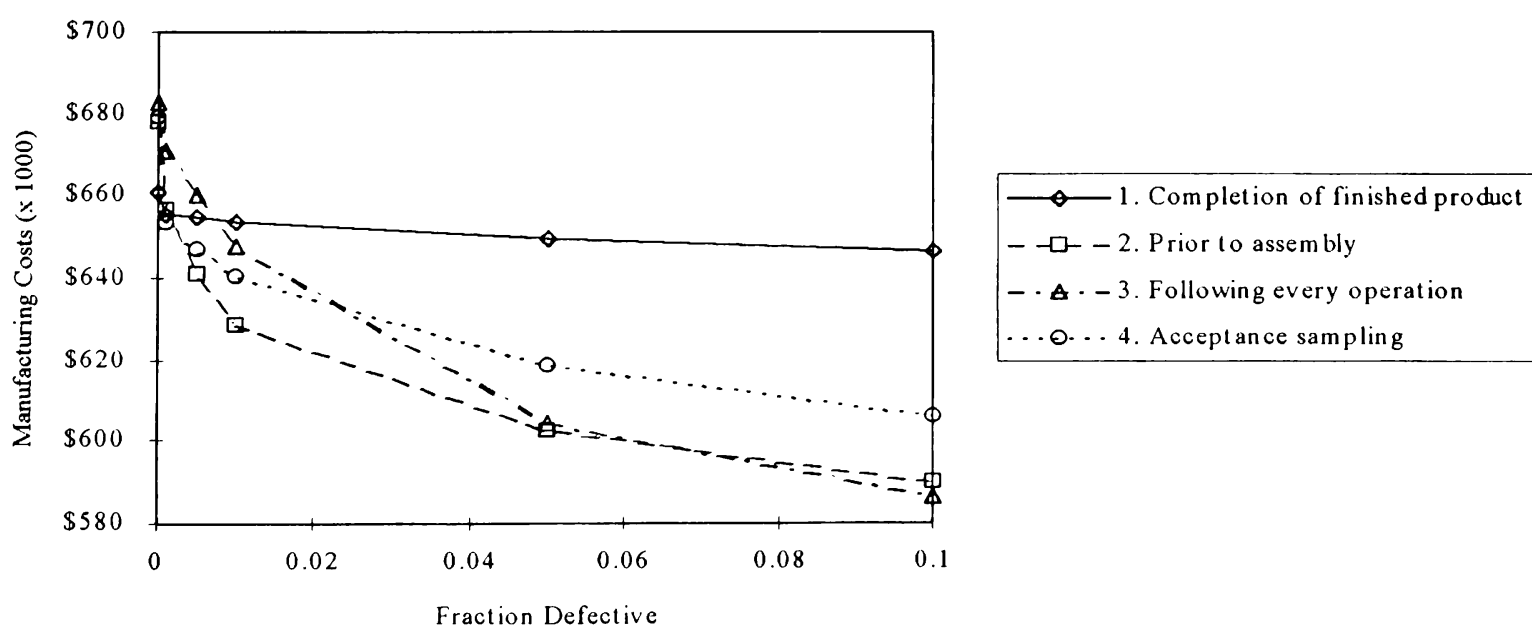

Figure 3: Manufacturing Costs 


\subsection{Profitability}

Profit is simply the difference between revenue and manufacturing costs. In these experiments, it is assumed that all product manufactured is sold. Thus, revenue is the product of the volume of finished product and the selling price. Table + lists the profit generated by each experiment. These results are sumniarized in the profitability curves of Figure 4 .

The profitability results support the value of reducing defective rates. For each of the inspection and defective removal strategies, the maximum profit occurs when the defective rate is zero. Morcover, the maximum profit at a zero defect rate occurs when inspection and defective removal is minimized. This agrees with Deming's third point which is to cease dependence on inspection and build quality into the process.

\section{CONCLUSIONS}

Simulation is a viable tool for assessing quality costs and evaluating quality improvement strategies. Quality costs can be defined in terms of the difference in profitability between the "as is" manufacturing system and the system after proposed improvements and defective rate reductions are made. These costs include lost profit, wasted materials, wasted resources, and wasted time in the production of defective parts. This waste can be quantified by conversion of simulation performance measures into costs and profits, without actually having to incur the cost of making the improvement. Quality improvement efforts can then be justified if the increase in profitability is sufficient to cover the costs of implementation.

\section{REFERENCES}

Campanella, J. 1990. Principles of quality costs, $2 \mathrm{~d}$ ed., ASQC Quality Press, Milwaukee, WI.

Gardner, L.L., M.E. Grant, and L.J. Rolston. 1992. Converting simulation data to comparative income statements. Proceedings of the 1992 Winter Simulation Conference, J.J. Swain, D. Goldsman, R.C. Crain, and J.R. Wilson, Eds., 13-16 Dec. 1992, Arlington, Virginia.

Table 4: Profit $(\times 1000)$

\begin{tabular}{|l|c|c|c|c|c|c|}
\hline \multicolumn{1}{|c|}{$\begin{array}{c}\text { Inspection and Defective } \\
\text { Removal Strategy }\end{array}$} & \multicolumn{5}{|c|}{ Defective Rate per Operation } \\
\cline { 2 - 7 } & $\mathbf{0 . 0 0 0}$ & $\mathbf{0 . 0 0 1}$ & $\mathbf{0 . 0 0 5}$ & $\mathbf{0 . 0 1 0}$ & $\mathbf{0 . 0 5 0}$ & $\mathbf{0 . 1 0 0}$ \\
\hline 1. Completion of finished product & $\$ 218$ & $\$ 173$ & $-\$ 5$ & $-\$ 175$ & $-\$ 605$ & $-\$ 6+4$ \\
\hline 2. Prior to assembly & $\$ 201$ & $\$ 213$ & $\$ 194$ & $\$ 138$ & $-\$ 84$ & $-\$ 286$ \\
\hline 3. Following every operation & $\$ 196$ & $\$ 199$ & $\$ 176$ & $\$ 120$ & $-\$ 86$ & $-\$ 282$ \\
\hline 4. Acceptance sampling & $\$ 199$ & $\$ 169$ & $\$ 152$ & $\$ 125$ & $-\$ 100$ & $-\$ 301$ \\
\hline
\end{tabular}

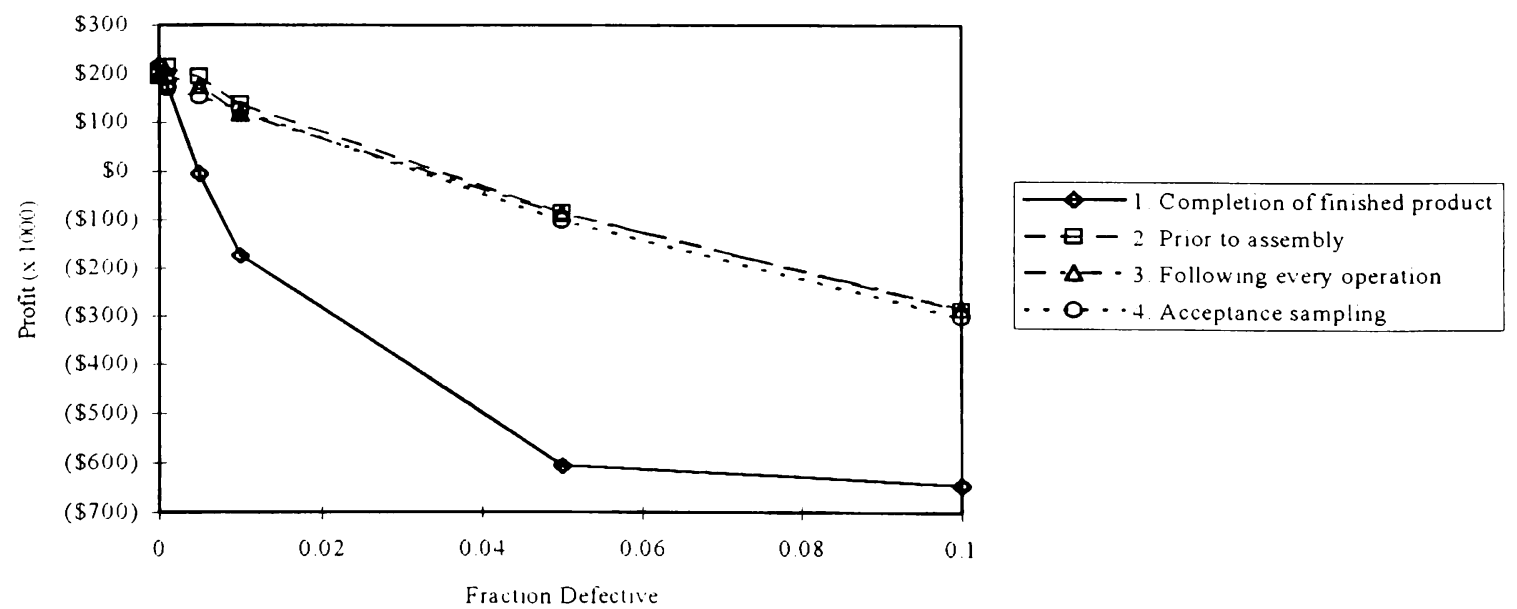

Figure 4: Profit 


\section{AUTHOR BIOGRAPHIES}

L. LESLIE GARDNER is an Assistant Professor at the University of Indianapolis with a joint appointment in the School of Business and the Department of Mathematics. She holds a B.A. degree in physics and mathematics from DePauw University, an M.S. degree in mathematics from Indiana State University, and M.S. and Ph.D. degrees in industrial enginecring from Purdue University. Her research interests are in graph theory, particularly decomposition of 3-connected graphs and partial 3-trees. She is also involved in simulation with costing. She is a member of APICS, ASQC, IIE, INFORMS, and the Mathematical Programming Society.

MARY E. GRANT is a Senior Consultant for Pritsker Corporation where she supports FI-2 and ARIS applications within the Enterprise Solutions Group. Previous work with Pritsker dealt with software development and applications of a variety of simulation packages. She was formerly a Scnior Analyst/ Programmer with Babcock \& Wilcox Aerospace Components Division where she applied simulation technology in a production scheduling environment for fabrication of aerospace components. She holds a B.S. degree in mathematics from Lincoln University and an M.S. degree in mathematics from Purdue University.

LAURIE J. ROLSTON is member of the Modeling and Information Sciences Lab at DowElanco where she specializes in the use of operations research techniques to improve R\&D and supply chain processes. She was formerly a consultant and software devcloper for Pritsker Corporation. While at Pritsker, she used simulation software for design analysis of manufacturing systems and for finite capacity scheduling. She is a co-author of the solutions manual to the third edition of Introduction to Simulation and SLAMI II. She holds a B.S.I.E. from Purdue University. 